\title{
Defective antioxidative activity of small dense HDL3 particles in type 2 diabetes: relationship to elevated oxidative stress and hyperglycaemia
}

Received: 27 May 2004 / Accepted: 11 October 2004 / Published online: 24 February 2005

C) Springer-Verlag 2005

\begin{abstract}
Aims/hypothesis: Elevated oxidative stress, hyperglycaemia, and dyslipidaemia involving low levels of HDL particles are key proatherogenic factors in type 2 diabetes mellitus. We examined the relationship of oxidative stress, and the degree of glycaemia and triglyceridaemia, to antioxidative function of HDL particle subspecies in type 2 diabetes. Subjects and methods: Five HDL subfractions $(2 \mathrm{~b}, 2 \mathrm{a}, 3 \mathrm{a}, 3 \mathrm{~b}, 3 \mathrm{c})$ were isolated by density gradient ultracentrifugation from well-controlled type 2 diabetic subjects $(n=20)$ and normolipidaemic, non-diabetic controls $(n=10)$. Specific antioxidative activity (capacity to protect LDL from oxidation on a unit particle mass or on a particle number basis), chemical composition and enzymatic activities were measured in each subfraction. Systemic oxidative stress was assessed as plasma levels of 8-isoprostanes. Results: Specific antioxidative activity of small dense HDL $3 b$ and $3 c$ particles in diabetic patients was significantly diminished (up to $-47 \%$, on a particle mass or particle number basis) as compared with controls. Plasma 8isoprostanes were markedly elevated (2.9-fold) in diabetic patients, were negatively correlated with both specific antioxidative activity of HDL3 subfractions and plasma HDL cholesterol (HDL-C) levels, and were positively correlated with glycaemia and triglyceridaemia. Paraoxonase 1 activity was consistently lower in diabetic HDL subfractions and was positively correlated with HDL3 antioxidative activity. The altered chemical composition of diabetic HDL3 subfractions (core cholesteryl ester depletion, triglyceride en-
\end{abstract}

\footnotetext{
E. Nobécourt · B. Hansel · S. Chantepie - M. J. Chapman A. Kontush $(\bowtie)$

Dyslipoproteinemia and Atherosclerosis Research, Unit 551, National Institute for Health and Medical Research (INSERM), Pavillon Benjamin Delessert, Hôpital de la Pitié, 83 boulevard de 1' Hôpital,

75651 Paris Cedex 13, France

e-mail: kontush@chups.jussieu.fr

Tel.: +33-1-42177976

Fax: +33-1-45828198
}

S. Jacqueminet $\cdot$ A. Grimaldi Diabetology Service, Hospital de la Pitié, Paris, France richment) was equally correlated with diminished antioxidative activity. Conclusions/interpretation: Antioxidative activity of small dense HDL is deficient in type 2 diabetes, is intimately linked to oxidative stress, glycaemia and hypertriglyceridaemia and primarily reflects abnormal intrinsic physicochemical properties of HDL particles.

Keywords Atherosclerosis - HDL subfractions - LDL oxidation - Oxidative stress - Plasma 8-isoprostanes · Type 2 diabetes

Abbreviations AAPH: 2,2'-azo-bis-(2-amidinopropane) hydrochloride - apo: apolipoprotein - C6NBD-PC: 1palmitoyl-2-6[7-nitrobenzoxadiazoxyl]aminocaproylphosphatidylcholine - CETP: cholesteryl ester transfer protein - CRP: C-reactive protein - FC: free cholesterol HDL-C: HDL cholesterol - LCAT: lecithin-cholesterol acyltransferase $\cdot$ PAF-AH: platelet-activating factor acetylhydrolase PL: phospholipid - PON: paraoxonase . REM: relative electrophoretic mobility $\cdot$ ROS: reactive oxygen species - sAA: specific antioxidative activity TBARS: thiobarbituric acid-reactive substances - TC: total cholesterol · TG: triglyceride

\section{Introduction}

Major cardiovascular risk factors implicated in accelerated atherosclerosis of type 2 diabetes mellitus include dyslipidaemia, hyperglycaemia, hypertension, visceral obesity and insulin resistance $[1,2]$. These risk factors act to induce an inflammatory state at the arterial wall, involving endothelial activation and oxidative stress $[1,2]$; the latter is expressed as a disequilibrium between elevated formation of reactive oxygen species (ROS) relative to defective plasma antioxidative capacity [3-5]. Under conditions of oxidative stress, ROS enhance formation of proinflammatory, oxidised LDL [6].

Atherogenic dyslipidaemia is a prominent feature of type 2 diabetes, and typically involves elevated levels of apolipoprotein B (apoB)-containing lipoproteins and subnormal 
levels of anti-atherogenic HDL $[2,7]$. HDL particles exert a spectrum of anti-atherogenic activities, which include potent antioxidative, anti-inflammatory, anti-thrombotic and anti-apoptotic actions [8]. Indeed, HDL particles can function as acceptors of oxidised lipids and equally possess the capacity to inactivate them by enzymatic and non-enzymatic mechanisms [9]. Enzymes associated with HDL particles, including paraoxonase $(\mathrm{PON})$ in its major isoform PON1, platelet-activating factor acetylhydrolase (PAF-AH) and lecithin-cholesterol acyltransferase (LCAT), can cleave oxidised lipids and thereby inhibit LDL oxidation. In addition, apoAI, the major HDL apolipoprotein, can remove oxidised lipids from LDL [9]. Finally, HDLs contain minor amounts of low-molecular-weight antioxidants, including vitamin E and carotenoids, as well as esterified polyunsaturated fatty acids; these components may modulate HDL antioxidative function $[10,11]$.

Hyperglycaemia, hyperinsulinaemia and hypertriglyceridaemia in type 2 diabetes lead to modification of HDL level, composition and function [7, 12-19]. Elevated plasma levels of triglyceride (TG)-rich lipoproteins drive cholesteryl ester transfer protein (CETP)-mediated heterotransfer of core lipids between TG-rich lipoproteins and HDLs, depleting HDLs of CE and enriching them in TG [7, 20]. As a result, both the conformation and function of apoAI may be altered [21], an effect that may be further enhanced by glycosylation of apoAI [14]. Such structural modifications are manifested in an attenuated capacity of apoAI to induce cellular cholesterol efflux and in accelerated HDL catabolism $[15,17]$.

Circulating HDL particles are heterogeneous in their physicochemical properties and anti-atherogenic activities [8]. Indeed, small dense HDLs inhibit expression of adhesion proteins by endothelial cells [22] and potently protect atherogenic LDLs against oxidative stress [23]. Equally, HDL2 from poorly controlled type 2 diabetic subjects displays decreased protection against macrophage-induced LDL oxidation [16].

The question arises then as to whether the potent antioxidative activities of small dense HDL3 are attenuated in type 2 diabetes. We provide evidence that small dense HDL3 particles exhibit defective antioxidative activity and display altered physicochemical properties in type 2 diabetic subjects under glycaemic control, and that such activity is intimately related to elevated oxidative stress and equally to the degree of both glycaemia and triglyceridaemia.

\section{Subjects and methods}

Subjects Type 2 diabetic subjects $(n=20)$ were recruited at the Diabetology Service, Pitie-Salpetrière Hospital (Paris, France). Type 2 diabetes was defined according to WHO criteria [24]. Inclusion criteria were as follows: (1) wellcontrolled diabetes $\left(\mathrm{HbA}_{1} \mathrm{c}<7.5 \%\right)$ under treatment with diet and/or oral hypoglycaemic drugs; (2) absence of insulin supplementation; (3) plasma TG and LDL cholesterol levels of less than $3.53(300 \mathrm{mg} / \mathrm{dl})$ and $4.14 \mathrm{mmol} / 1$ $(160 \mathrm{mg} / \mathrm{dl})$ respectively; (4) absence of hypertension (systolic blood pressure $<140 \mathrm{mmHg}$ and diastolic blood pressure $<90 \mathrm{mmHg}$ ) with or without hypotensive treatment; and (5) BMI of less than $30 \mathrm{~kg} / \mathrm{m}^{2}$. Normolipidaemic, nondiabetic, age-matched healthy controls $(n=10)$ were recruited in the general population in the same area. The null hypothesis stated that HDL antioxidative activity does not differ between the diabetic group and the control group. A group size of 25 subjects (15 patients with type 2 diabetes and ten controls) was calculated in order to detect significant differences of $30 \%$ or more in HDL antioxidative activity between the groups at the level of $p<0.05$ (statistical power 0.80 ). All subjects gave written informed consent. The clinical protocol was approved by the institutional ethics committee.

Medications taken by diabetic patients were as follows: sulphonylurea (ten subjects), biguanides (17 subjects), angiotensin-converting enzyme inhibitors (two subjects) and calcium channel blockers (one subject). Diabetic and control groups were matched for sex; there were five $(25 \%)$ and two $(20 \%)$ female subjects in the two groups, respectively. Women were postmenopausal and were not receiving oestrogen therapy. Antioxidative and enzymatic properties of HDL subfractions did not differ between male and female subjects within each group (data not shown). All subjects were non-smokers and either abstainers or moderate alcohol consumers (less than $25 \mathrm{~g} /$ day); none presented symptomatic cardiovascular disease or renal, hepatic, gastrointestinal, pulmonary, endocrine or oncological disease, or received antioxidant vitamins, glitazones or drugs known to affect lipid metabolism. As a marker of vitamin intake, plasma vitamin $\mathrm{E}$ was assayed in a subgroup of ten patients and five controls using HPLC with ultraviolet detection [25]; no difference between the groups was observed (data not shown). As a marker of inflammation, high-sensitivity C-reactive protein (CRP) was measured by immunonephelometry (inter- and intra-assay coefficients of variation $<5 \%$ ) [26].

Blood samples Venous blood was collected after an overnight fast. EDTA plasma and serum were immediately isolated, mixed with sucrose (final concentration $0.6 \%$ ) as a cryoprotectant for lipoproteins [27], and frozen at $-80^{\circ} \mathrm{C}$ under nitrogen.

Isolation of lipoproteins Lipoproteins were fractionated by single-spin equilibrium density gradient ultracentrifugation [28] for $48 \mathrm{~h}$ at $40,000 \times g$ in a SW41-Ti rotor (Beckman Coulter, Pasadena, CA, USA) at $4^{\circ} \mathrm{C}$. Five HDL subfractions were isolated: large, light HDL2b (density 1.063$1.087 \mathrm{~g} / \mathrm{ml}$ ) and $2 \mathrm{a}$ particles (density $1.088-1.110 \mathrm{~g} / \mathrm{ml}$ ), and small dense HDL3a (density 1.110-1.129 g/ml), 3b (density $1.129-1.154 \mathrm{~g} / \mathrm{ml}$ ) and 3c particles (density 1.154 $1.170 \mathrm{~g} / \mathrm{ml})[28,29]$. The validity and reproducibility of this density fractionation of HDL particle subspecies have been extensively documented [28-30]. Reference LDLs were isolated using the same procedure from one healthy, normolipidaemic male donor for all oxidation experiments. After dialysis against Dulbecco's PBS (pH 7.4) for $24 \mathrm{~h}$ at 
$4^{\circ} \mathrm{C}$ to remove EDTA and $\mathrm{KBr}$, lipoproteins were maintained at $4^{\circ} \mathrm{C}$ and used within 1 week.

Characterisation of lipoproteins Total cholesterol (TC), free cholesterol (FC), phospholipid (PL) and TG concentrations were measured using commercially available kits (CHOP-PAP, Biomerieux, France). CE content was calculated by multiplying the difference between TC and FC by 1.67 [28]. Total protein was measured using the BCA assay [28]. Total lipoprotein mass was calculated as the sum of the mass of total protein, CE, FC, PL and TG. ApoAI and apoAII were quantified by immunonephelometry [23]. Inter- and intra-assay coefficients of variation for lipoprotein mass were less than 5\% [30].

Molecular weights of HDL subfractions were calculated by transforming concentration data $(\mathrm{mg} / \mathrm{dl})$ into absolute molar units using $M_{\mathrm{r}}$ of CE, FC, PL and TG of 650,387 , 750 and 850 respectively [31]. The HDL protein moiety was considered to consist of two apolipoproteins, apoA-I and apoA-II, and the molecular weight of the protein moiety in each HDL subfraction was calculated using the total protein content $(\mathrm{mg} / \mathrm{dl})$ converted to molarity on the basis of relative mass content of apoA-I and apoA-II.

Enzymatic activities of HDL subfractions PON1 activity was determined photometrically at $270 \mathrm{~nm}$ as arylesterase activity using phenyl acetate as a substrate [23]. PAF-AH activity was measured using a fluorescent substrate, 1Palmitoyl-2-6[7-nitrobenzoxadiazoxyl]aminocaproyl-phosphatidylcholine (C6NBD-PC) [32]. LCAT activity was measured as PL hydrolysing activity using a fluorescent LCAT activity kit (Roar Biomedical, New York, NY, USA) [33]. Inter- and intra-assay coefficients of variation were 2.2 and $9.8 \%$ for PON1 activity, 5.1 and $8.6 \%$ for PAF-AH activity and 5.1 and $6.5 \%$ for LCAT activity measurements, respectively.

Antioxidative activity of HDL subfractions Reference LDL (TC, $0.26 \mathrm{mmol} / 1$, equivalent to $10 \mathrm{mg} / \mathrm{dl}$ ) was incubated at $37^{\circ} \mathrm{C}$ in PBS in the presence of a water-soluble azo-initiator of oxidation, 2,2'-azo-bis-(2-amidinopropane) hydrochloride (AAPH; $1 \mathrm{mmol} / \mathrm{l})$. Individual HDL subfractions were added to LDL directly before oxidation. The PBS was treated with Chelex 100 ion exchange resin (BioRad, Marnes-la-Coquette, France) for $1 \mathrm{~h}$ to remove contaminating transition metal ions. Accumulation of conjugated dienes was continuously measured as the increment in absorbance at $234 \mathrm{~nm}$ [23]; two characteristic phases were identified, the lag phase and the propagation phase. To characterise the oxidation kinetics, average oxidation rates within each phase, the duration of each phase and maximal amount of dienes formed were calculated for each absorbance curve. In addition, thiobarbituric acid-reactive substances (TBARS) were measured after $24 \mathrm{~h}$ of oxidation, as described elsewhere [23].

For each HDL subfraction, specific antioxidative activity (sAA) was determined on a unit particle mass basis for each subfraction $(0.1 \mathrm{~g}$ total mass $/ 1)$ at an HDL:LDL ratio within the physiological range of 2 to $6 \mathrm{~mol} / \mathrm{mol}$, in order to assess the intrinsic capacity of HDL subfractions to protect LDL from oxidation [34]. Equally, sAA values were recalculated on the basis of particle number using molar concentrations of HDL subfractions obtained using HDL molecular weights.

In separate experiments, control and diabetic LDL (TC, $0.26 \mathrm{mmol} / \mathrm{l})$ were oxidised by AAPH $(1 \mathrm{mmol} / \mathrm{l})$ in the absence of HDL subfractions.

Plasma markers of oxidative stress 8-Isoprostanes, products of non-enzymatic oxidation of arachidonic acid in vivo that represent a robust marker of systemic oxidative stress [3], were measured in plasma $(1 \mathrm{ml})$ by a commercial ELISA assay after purification (Cayman Chemical, Ann Arbor, MI, USA; inter- and intra-assay coefficients of variation 8.0 and $11.1 \%$, respectively).

Statistical analysis Between-group differences in continuous variables were analysed by Student's two-tailed $t$-test for independent groups. Differences in dichotomous variables were analysed by Fisher's exact test. Pearson's moment-product correlation coefficients were calculated to evaluate relationships between variables. All results are expressed as means \pm SD. A $p$ value of less than 0.05 was considered statistically significant.

\section{Results}

Biological and clinical characteristics of type 2 diabetic patients and non-diabetic control subjects Type 2 diabetic subjects were dyslipidaemic as indicated by significantly elevated plasma levels of TG (2.2-fold, $p<0.01)$ and lower levels of both HDL cholesterol (HDL-C; $-19 \%, p<0.01$ ) and apoAI $(-15 \%, p<0.01)$ as compared with controls. While LDL cholesterol levels were similar in the two groups, apoB concentrations tended to be elevated $(+17 \%)$ in diabetic subjects, thereby reflecting increased concentrations of TG-rich particles (Table 1). Furthermore, the ratio of TC:HDL-C was significantly higher in diabetic subjects ( $4.40 \pm 1.03$ vs $3.45 \pm 0.72, p<0.05)$, indicative of an atherogenic lipid profile.

Plasma TG and HDL-C concentrations were negatively correlated $(r=-0.65, p<0.001)$. Glycaemia was well controlled $\left(\mathrm{HbA}_{1} \mathrm{c} 7.0 \pm 1.1 \%\right)$; nonetheless, mean fasting glucose concentration was elevated $(+43 \%, p<0.01)$ in the diabetic group (Table 1). Finally, our non-obese diabetic subjects exhibited a higher BMI relative to controls.

Systemic oxidative stress and inflammation Systemic oxidative stress assessed as plasma levels of 8-isoprostanes was 2.9-fold higher in type 2 diabetic subjects than in controls (Table 1). Levels of 8-isoprostanes were positively correlated with plasma levels of $\mathrm{HbA}_{1} \mathrm{c}$, glucose and TC: HDL-C ratio $(r=0.52, p<0.05 ; r=0.41, p<0.05$; and $r=0.42$, $p<0.05$, respectively) and were negatively correlated with HDL-C levels $(r=-0.46, p<0.05)$ (Fig. 1). These correlations did not persist separately in the study groups, indicating that they were rather related to between-group 
Table 1 Clinical and biological characteristics of type 2 diabetic patients and normolipidaemic, non-diabetic control subjects

\begin{tabular}{|c|c|c|}
\hline & $\begin{array}{l}\text { Control subjects } \\
(n=10)\end{array}$ & $\begin{array}{l}\text { Type } 2 \text { diabetes } \\
\text { patients }(n=20)\end{array}$ \\
\hline Age (years) & $54.7 \pm 11.7$ & $57.0 \pm 11.1$ \\
\hline BMI $\left(\mathrm{kg} / \mathrm{m}^{2}\right)$ & $23.0 \pm 1.8$ & $27.7 \pm 2.4 * * *$ \\
\hline $\operatorname{Sex}(M / F)$ & $8 / 2$ & $15 / 5$ \\
\hline $\mathrm{HbA}_{1} \mathrm{c}(\%)$ & $5.1 \pm 0.1$ & $7.0 \pm 1.1 * *$ \\
\hline \multicolumn{3}{|l|}{ Plasma parameters } \\
\hline Fasting glucose $\mathrm{e}^{\mathrm{a}}$ & $5.37 \pm 0.32$ & $7.69 \pm 2.08 * *$ \\
\hline $\mathrm{TG}^{\mathrm{a}}$ & $0.86 \pm 0.26$ & $1.90 \pm 1.13 * *$ \\
\hline $\mathrm{TC}^{\mathrm{a}}$ & $5.09 \pm 0.69$ & $5.22 \pm 0.83$ \\
\hline LDL cholesterol $^{\mathrm{a}}$ & $3.18 \pm 0.71$ & $3.20 \pm 0.71$ \\
\hline ApoB $100^{b}$ & $1.38 \pm 0.27$ & $1.61 \pm 0.38$ \\
\hline HDL cholesterol $^{\mathrm{a}}$ & $1.51 \pm 0.21$ & $1.22 \pm 0.20 * *$ \\
\hline ApoA-I ${ }^{\mathrm{b}}$ & $51.6 \pm 4.5$ & $43.6 \pm 6.2 * *$ \\
\hline 8-Isoprostanes ${ }^{\mathrm{c}}$ & $102 \pm 54$ & $291 \pm 194 *$ \\
\hline
\end{tabular}

ApoAI and apoB100 were measured in a subset of eight controls and 11 diabetic patients

$* p<0.05$

$* * p<0.01$

$* * * p<0.001$ vs controls

${ }^{\mathrm{a}} \mathrm{mmol} / 1$

${ }^{\mathrm{b}} \mu \mathrm{mol} / \mathrm{l}$

${ }^{\mathrm{c}} \mathrm{pmol} / 1$

differences in 8-isoprostane levels. The degree of inflammation, evaluated as serum CRP, was similar in diabetic (median $40 \mathrm{nmol} / \mathrm{l}$, range 9-192) and control (median 42 nmol/l, range 8-64) subjects.

Quantitative and qualitative features of HDL subfractions Molar concentrations and molecular weights of small HDL3 subfractions did not differ significantly between the groups (Table 2); molar concentrations of large HDL2b and 2 a subfractions tended to be lower $(-11$ and $-5 \%$, respectively) in diabetic patients as compared with controls.

HDL subfractions from diabetic subjects were distinct in chemical composition, being depleted in $\mathrm{CE}$ and $\mathrm{FC}$ and enriched in TG and PL as compared with controls, consistent with previously reported data [13]. Core CE content was significantly lower in all diabetic HDL subfractions as compared with controls. In contrast, the proportion of TG in diabetic HDL subfractions was consistently higher. In addition, FC content was significantly lower in diabetic HDL2a, 3a and $3 \mathrm{~b}$ subfractions relative to the control group (Table 2). In addition, PL content was significantly higher in diabetic HDL2b, 2a, 3a and 3b subfractions and total protein was significantly higher in the diabetic HDL3c subfraction as compared with controls (Table 2). In contrast, absolute levels of apoAI and apoAII in HDL subfractions did not differ significantly between diabetic and control groups (data not shown). Plasma levels of 8-isoprostanes correlated negatively with cholesterol content of HDL subfractions, specifically with \%CE content in HDL $3 b$ and $3 \mathrm{c}$ subfractions and with $\% \mathrm{FC}$ content in HDL2a and $3 \mathrm{a}$ subfractions (data not shown).
Oxidative protection of $L D L$ by $H D L$ subfractions Consistent with our recent data in normolipidaemic subjects [23], small dense HDL subfractions from both groups delayed AAPH-induced oxidation of reference LDL under conditions employed to measure sAA (Fig. 2a, b). When expressed on a particle mass basis, the sAA of small dense HDL $3 b$ and $3 c$ subfractions was significantly decreased $(-19$ to $-34 \%, p<0.05)$ in patients with type 2 diabetes as compared with controls (Fig. 2c, d). In diabetic subjects, only HDL3c particles significantly decreased LDL oxidation rate in the propagation phase $(-39 \%, p<0.001)$; none of the HDL subfractions significantly prolonged this phase. In control subjects, all three small dense HDL $3 a, 3 b$ and $3 c$ subfractions significantly decreased LDL oxidation rate in the propagation phase: HDL3a $(-13 \%, p<0.01)<$ HDL3b $(-37 \%, p<0.001)<$ HDL3c $(-58 \%, p<0.001)$. In addition, HDL $3 \mathrm{~b}$ and $3 \mathrm{c}$ significantly prolonged this phase: HDL3b $(+28 \%, p<0.001)<$ HDL3c $(+47 \%, p<0.001)$ (Fig. 2$)$.

When calculated on a particle number basis, the sAA of diabetic small dense HDL3 subfractions was again significantly impaired. With each subfraction at a concentration
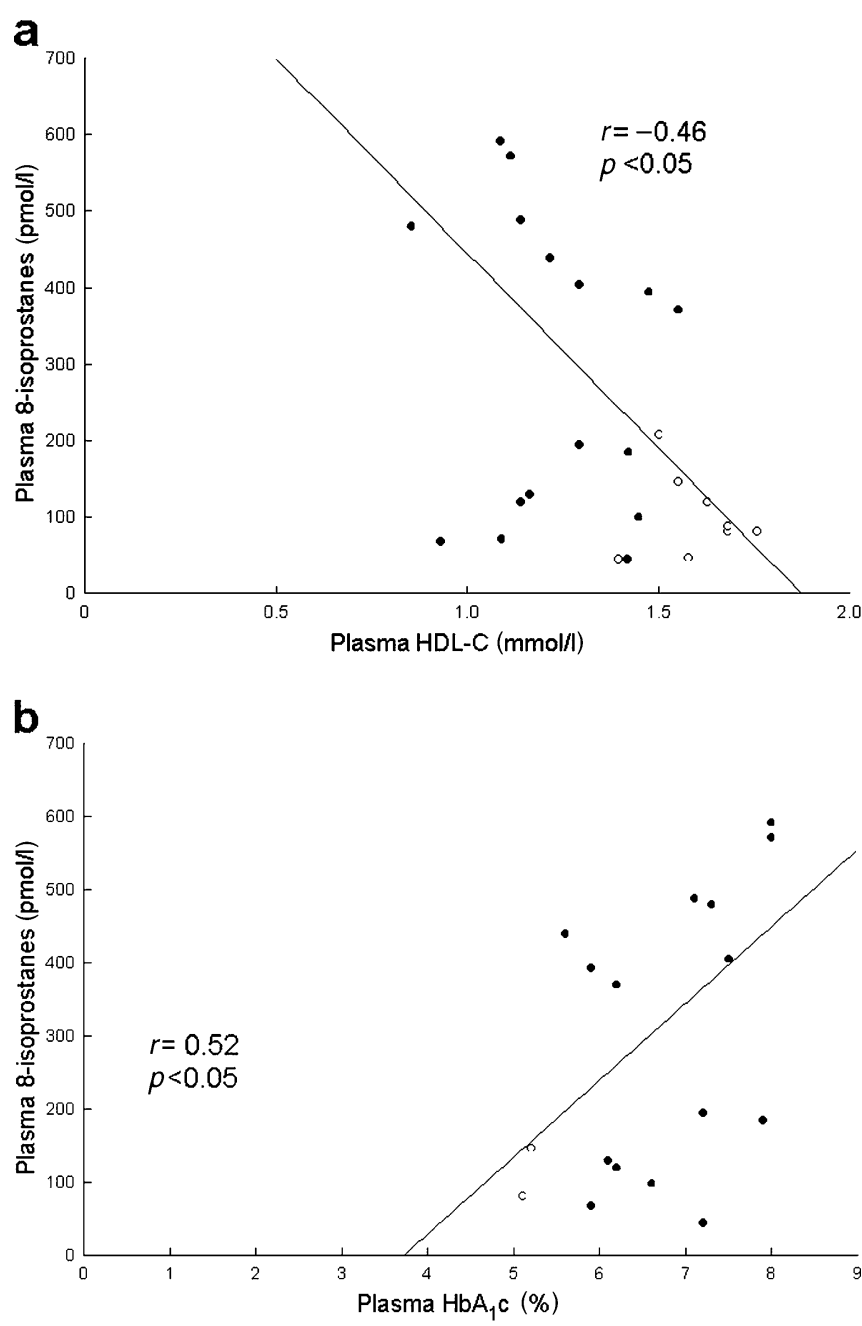

Fig. 1 Correlations between systemic oxidative stress assessed as plasma 8-isoprostane levels and concentrations of a HDL-C and $\mathbf{b}$ $\mathrm{HbA}_{1} \mathrm{c}$ in patients with type 2 diabetes (filled circles) and control subjects (open circles) 
Table 2 Molar concentration, molecular weight, chemical composition of lipids and protein (w/w) and enzymatic activities of HDL subfractions in type 2 diabetic patients $(n=20)$ and control subjects $(n=10)$

\begin{tabular}{|c|c|c|c|c|c|c|}
\hline & Group & HDL2b & HDL2a & HDL3a & HDL3b & HDL3c \\
\hline \multirow{2}{*}{$\begin{array}{l}\text { Molar concentration } \\
(\mu \mathrm{mol} / \mathrm{l})\end{array}$} & Type 2 diabetes & $1.67 \pm 0.53$ & $1.94 \pm 0.35$ & $1.96 \pm 0.26$ & $1.50 \pm 0.26$ & $1.10 \pm 0.16$ \\
\hline & Control & $1.87 \pm 0.63$ & $2.04 \pm 0.41$ & $1.96 \pm 0.48$ & $1.54 \pm 0.56$ & $1.03 \pm 0.43$ \\
\hline \multirow{2}{*}{$\begin{array}{l}\text { Molecular weight } \\
\left(M_{\mathrm{r}} 10_{3}\right)\end{array}$} & Type 2 diabetes & $385 \pm 56$ & $372 \pm 37$ & $345 \pm 26$ & $199 \pm 33$ & $147 \pm 21$ \\
\hline & Control & $424 \pm 99$ & $380 \pm 80$ & $350 \pm 76$ & $194 \pm 36$ & $161 \pm 36$ \\
\hline \multirow[t]{2}{*}{$\mathrm{FC}$} & Type 2 diabetes & $5.9 \pm 2.3$ & $3.1 \pm 0.7 * *$ & $2.4 \pm 0.5 * *$ & $2.2 \pm 0.5^{*}$ & $2.3 \pm 0.7$ \\
\hline & Control & $6.9 \pm 1.9$ & $4.4 \pm 1.1$ & $3.2 \pm 0.7$ & $2.6 \pm 0.6$ & $2.2 \pm 0.7$ \\
\hline \multirow[t]{2}{*}{$\mathrm{CE}$} & Type 2 diabetes & $21.6 \pm 4.0 * * *$ & $19.9 \pm 3.2 * * *$ & $19.4 \pm 2.9 * * *$ & $16.4 \pm 2.6^{* * *}$ & $12.7 \pm 3.8^{* * *}$ \\
\hline & Control & $29.1 \pm 5.3$ & $26.6 \pm 6.7$ & $25.2 \pm 5.4$ & $22.5 \pm 3.4$ & $21.0 \pm 3.2$ \\
\hline \multirow[t]{2}{*}{ TG } & Type 2 diabetes & $9.2 \pm 6.3$ & $5.5 \pm 3.1$ & $4.6 \pm 2.8$ & $6.0 \pm 5.7$ & $5.3 \pm 6.2$ \\
\hline & Control & $6.9 \pm 3.6$ & $3.9 \pm 1.3$ & $3.5 \pm 1.0$ & $3.5 \pm 2.4$ & $4.4 \pm 3.7$ \\
\hline \multirow[t]{2}{*}{ PL } & Type 2 diabetes & $27.2 \pm 5.0^{*}$ & $31.4 \pm 2.6^{* *}$ & $29.4 \pm 1.9 * *$ & $20.7 \pm 2.0^{*}$ & $10.6 \pm 2.6$ \\
\hline & Control & $20.0 \pm 9.8$ & $23.9 \pm 11.4$ & $22.6 \pm 10.5$ & $15.9 \pm 8.2$ & $9.8 \pm 7.0$ \\
\hline \multirow[t]{2}{*}{ Protein } & Type 2 diabetes & $36.0 \pm 4.0$ & $40.0 \pm 3.5$ & $44.1 \pm 2.9$ & $54.7 \pm 5.4$ & $69.1 \pm 7.2 *$ \\
\hline & Control & $37.2 \pm 5.5$ & $41.3 \pm 5.7$ & $45.5 \pm 5.8$ & $55.5 \pm 6.7$ & $62.5 \pm 8.1$ \\
\hline \multirow[t]{2}{*}{$\mathrm{PON}^{\mathrm{a}}$} & Type 2 diabetes & $30 \pm 21$ & $58 \pm 45$ & $145 \pm 105^{*}$ & $1021 \pm 675$ & $2423 \pm 939$ \\
\hline & Control & $40 \pm 21$ & $96 \pm 73$ & $267 \pm 198$ & $1259 \pm 848$ & $2653 \pm 1137$ \\
\hline \multirow[t]{2}{*}{ PAF-AH ${ }^{b}$} & Type 2 diabetes & $3.1 \pm 1.0$ & $1.0 \pm 0.4$ & $0.9 \pm 0.6$ & $1.7 \pm 0.7$ & $2.7 \pm 0.7^{*}$ \\
\hline & Control & $2.3 \pm 1.1$ & $1.0 \pm 0.8$ & $1.0 \pm 0.5$ & $2.1 \pm 0.4$ & $3.3 \pm 0.5$ \\
\hline \multirow[t]{2}{*}{ LCAT $^{\mathrm{c}}$} & Type 2 diabetes & $10.9 \pm 8.5$ & $9.5 \pm 6.5$ & $8.9 \pm 8.3$ & $15.1 \pm 9.1 *$ & $21.5 \pm 12.6$ \\
\hline & Control & $5.6 \pm 6.3$ & $5.8 \pm 6.9$ & $2.6 \pm 2.1$ & $3.6 \pm 4.5$ & $13.4 \pm 13.8$ \\
\hline
\end{tabular}

$* p<0.05$

$* * p<0.01$

$* * * p<0.001$ vs controls

anmol of 4-nitrophenol formed per min per mg protein

${ }^{b}$ mol of C6NBD released per min per mg protein

${ }^{c}$ Percentage of hydrolysed fluorescent PL substrate

of $1 \mu \mathrm{mol} / \mathrm{l}$, HDL3b and 3c particles from diabetic patients were less potent in attenuating LDL propagation rate as compared with controls $(-23 \%, p<0.05$, and $-36 \%, p<$ 0.01 , respectively; Fig. 3); corresponding differences in the prolongation of the propagation phase were -33 and $-43 \%$ $(p<0.05)$.

Consistent with these results, accumulation of TBARS, a marker of advanced lipid breakdown products, was significantly higher in the presence of HDL3b and HDL3c subfractions from diabetic patients $(+41$ and $+47 \%$, respectively, $p<0.05$ ) as compared with controls (data not shown).

HDL-mediated protection of LDL was most pronounced at late stages of oxidation (Fig. 2a, b), indicating that antioxidative activity of HDL subfractions was primarily associated with inactivation of lipid hydroperoxides [23]. Nevertheless, HDL3c subfractions from both diabetic and control groups significantly prolonged the lag phase of LDL oxidation $(+39$ and $+63 \%$, respectively). The difference between the two groups was not significant. It is also noteworthy that the apparent increase in maximal diene formation observed in the presence of diabetic and control HDL2b, 2a and 3a subfractions (Fig. 2a, b) was due to the contribution from oxidation of HDL subfractions themselves [23].

When LDLs from diabetic and control subjects were oxidised in the absence of HDL subfractions, no significant difference in any oxidation parameter was observed be- tween the groups (data not shown), as reported earlier in patients with type 2 diabetes [35-37].

Plasma 8-isoprostanes were positively correlated with LDL oxidation rate in the propagation phase in the presence of HDL3a $(r=0.51, p<0.05)$, HDL3b $(r=0.68, p<0.001)$ and HDL3c $(r=0.80, p<0.001$; Fig. 4$)$ subfractions and were negatively correlated with duration of the propagation phase in the presence of HDL3a $(r=-0.41, p<0.05)$, HDL3b $(r=$ $-0.50, p<0.05)$ and HDL3c $(r=-0.49, p<0.05)$ subfractions and were positively correlated with TBARS accumulation in the presence of HDL $3 \mathrm{~b}(r=0.61, p<0.01)$ and HDL3c $(r=0.51, p<0.05)$ subfractions. These correlations persisted in diabetic, but not in control, subjects (data not shown). Correlations of comparable significance were calculated when SAA was expressed on a particle number basis (data not shown).

Fasting levels of glucose positively correlated with LDL oxidation rate in the presence of HDL3b $(r=0.53, p<0.01)$ and HDL $3 \mathrm{c}(r=0.55, p<0.01)$ subfractions and with TBARS accumulation in the presence of HDL3b $(r=0.41, p<0.05)$ and HDL $3 c(r=0.48, p<0.05)$ subfractions. Plasma levels of TG positively correlated with LDL oxidation rate in the presence of HDL3b $(r=0.38, p<0.05)$ and HDL3c $(r=0.39$, $p<0.05)$ subfractions. These correlations persisted in diabetic, but not in control, subjects (data not shown). Finally, negative correlations were observed between plasma levels of glucose and TG and the duration of LDL propagation phase in the presence of HDL3c (data not shown) particles. 
a

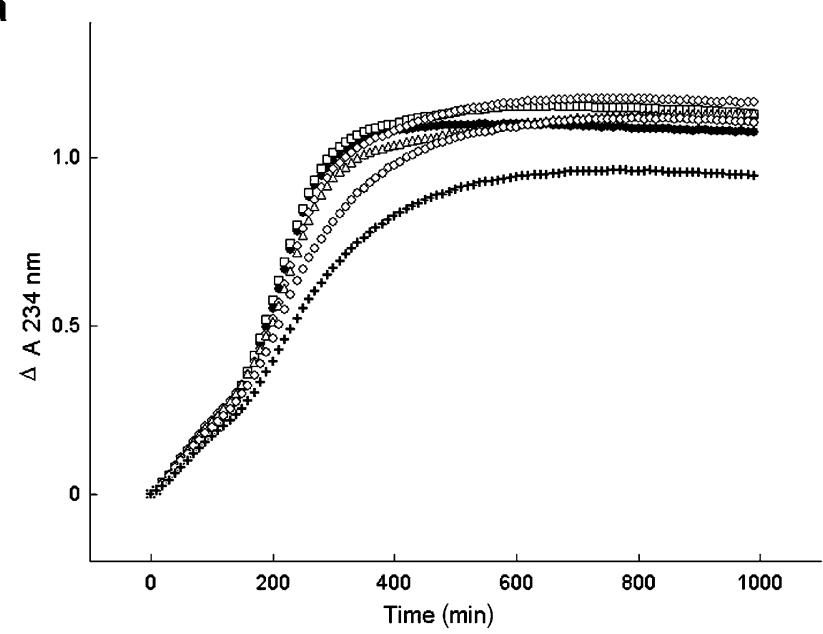

C

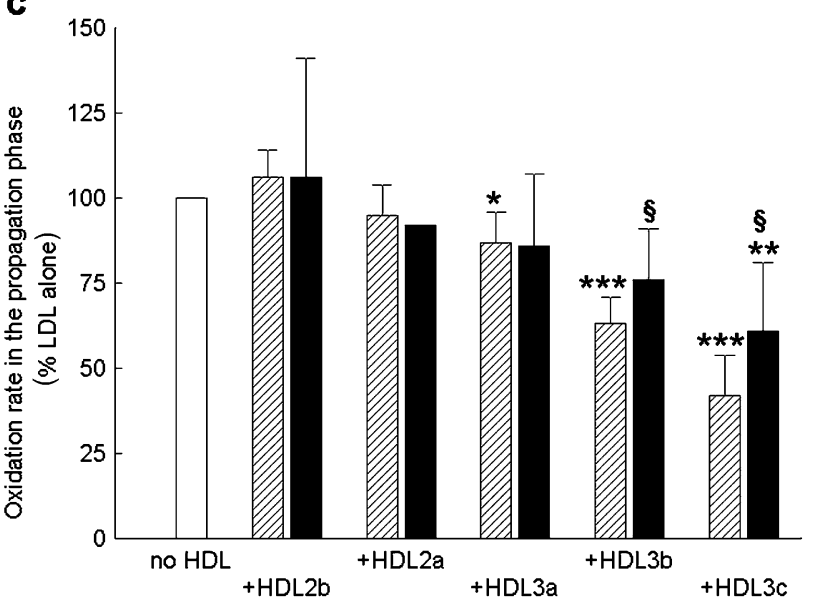

Fig. 2 Specific antioxidant activity: influence of HDL subfractions isolated from patients with type 2 diabetes and from control subjects on LDL oxidation. Oxidation kinetics were averaged for LDL incubated in the absence of HDL (filled circles) or in the presence of HDL2b (squares), 2a (diamonds), 3a (triangles), $3 \mathrm{~b}$ (circles) and $3 \mathrm{c}$

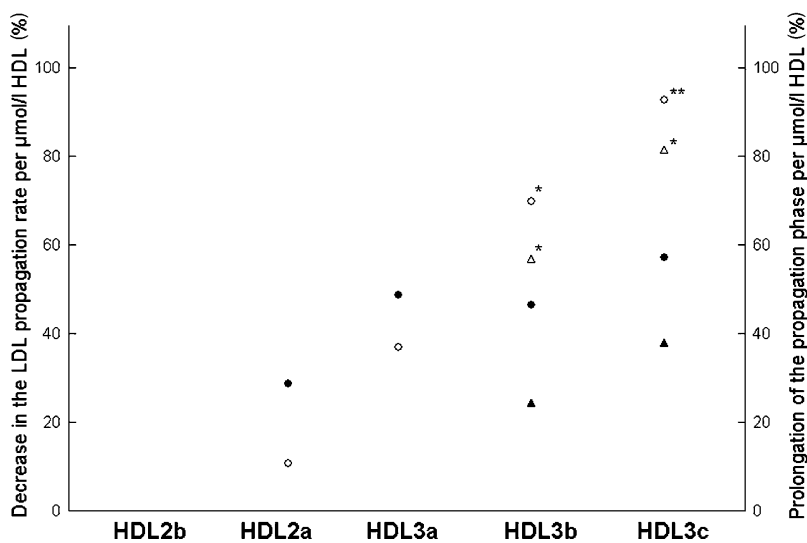

Fig. 3 Antioxidant activity of HDL subfractions calculated per 1.0 $\mu \mathrm{mol} / 1$ of each subfraction. Inhibition by HDL subfractions of LDL oxidation in the propagation phase is shown as mean percentage decrease in the oxidation rate (left axis; circles) and as mean percentage prolongation of the phase (right axis; triangles) for both patients with type 2 diabetes (filled symbols) and control subjects (open symbols). Negative values occasionally calculated for light HDL subfractions are not shown. ${ }^{* *} p<0.01, * p<0.05$ vs corresponding parameter in the diabetic group
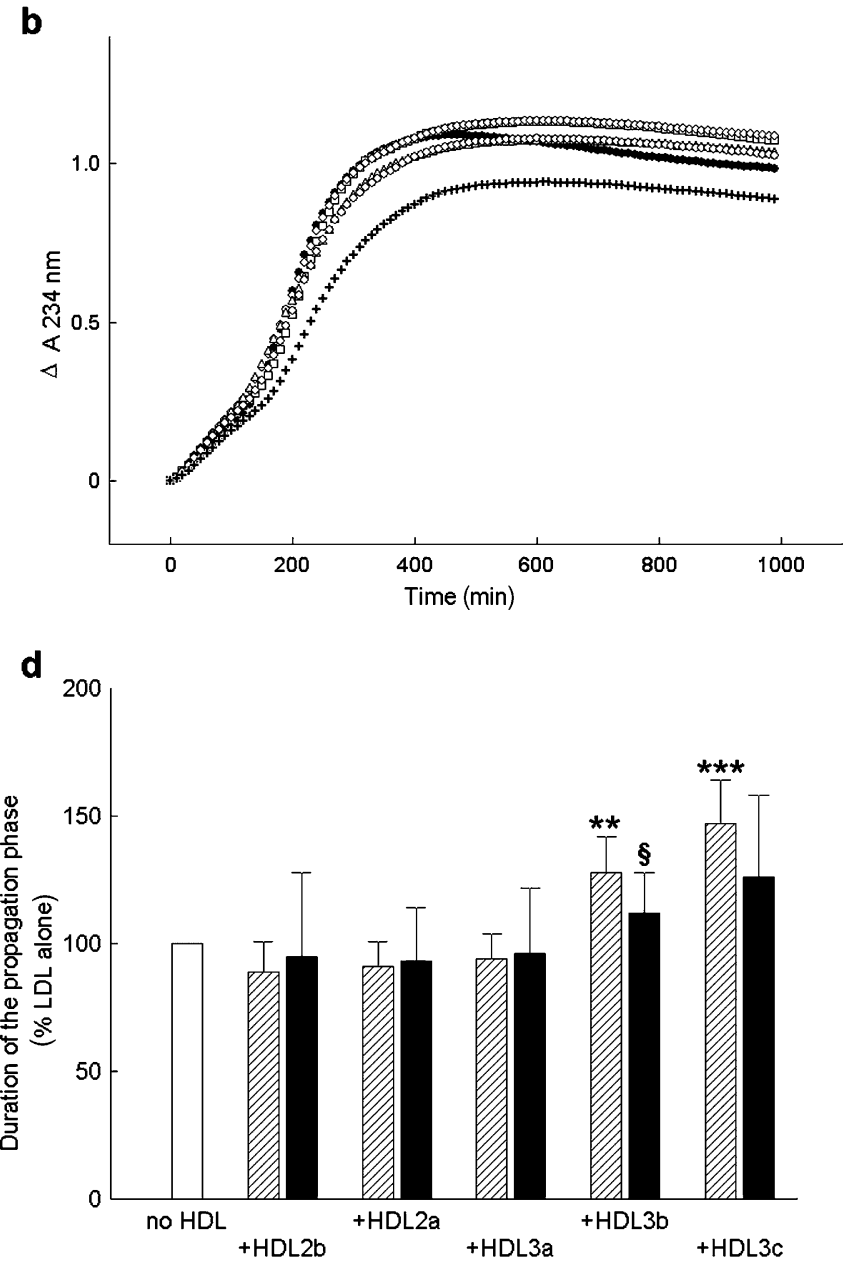

(crosses) for a all control subjects and $\mathbf{b}$ all diabetic patients. LDL oxidation rate in the propagation phase (c) and the duration of the propagation phase (d) are shown for control subjects (hatched bars) and diabetic patients (filled bars). ${ }^{* * *} p<0.001,{ }^{* *} p<0.01,{ }^{*} p<0.05$ vs incubation without added HDL; ${ }^{\S} p<0.05$ vs control subjects

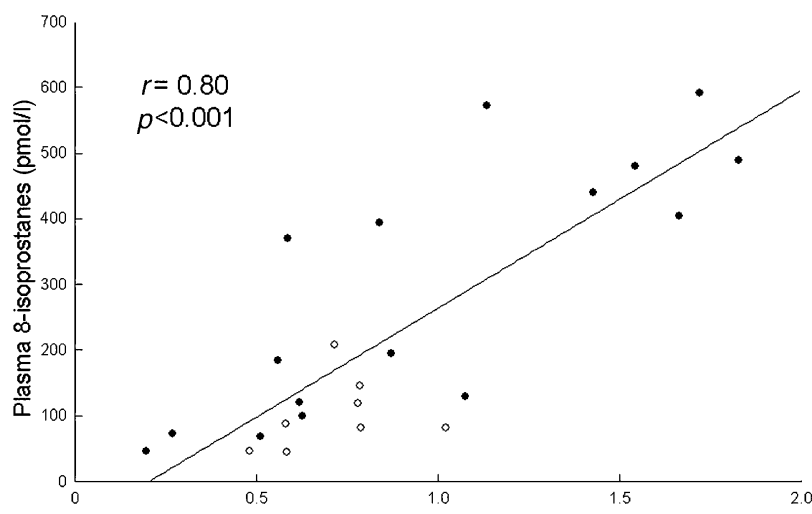

$\mathrm{LDL}$ propagation rate in the presence of $\mathrm{HDL} 3 \mathrm{c}$ (mol dienes per [mol LDL/min])

Fig. 4 Correlation between systemic oxidative stress assessed as plasma levels of 8-isoprostanes and LDL oxidation rate in the propagation phase in the presence of HDL3c in patients with type 2 diabetes (filled circles) and control subjects (open circles) 
Table 3 Correlations between activities of PON, PAF-AH and LCAT and parameters of LDL oxidation measured in the presence of all HDL subfractions from diabetic and control subjects

\begin{tabular}{|c|c|c|c|c|}
\hline & \multicolumn{4}{|c|}{ Correlation coefficients } \\
\hline & $\begin{array}{l}\text { Duration } \\
\text { of the } \\
\text { lag phase }\end{array}$ & $\begin{array}{l}\text { Oxidation } \\
\text { rate in the } \\
\text { propagation } \\
\text { phase }\end{array}$ & $\begin{array}{l}\text { Duration } \\
\text { of the } \\
\text { propagation } \\
\text { phase }\end{array}$ & $\begin{array}{l}\text { Maximal } \\
\text { amount } \\
\text { of dienes }\end{array}$ \\
\hline $\begin{array}{l}\text { PON } \\
\text { activity }\end{array}$ & $0.20 * * *$ & $-0.62 * * *$ & $0.60 * * *$ & $-0.51 * * *$ \\
\hline $\begin{array}{c}\text { PAF-AH } \\
\text { activity }\end{array}$ & $0.21^{*}$ & -0.01 & 0.10 & -0.02 \\
\hline $\begin{array}{l}\text { LCAT } \\
\text { activity }\end{array}$ & 0.18 & $-0.23 *$ & $0.22 *$ & $-0.34 * *$ \\
\hline
\end{tabular}

Protein components of HDL possessing antioxidative activity PON1 activity (assayed with phenyl acetate as substrate) was consistently lower (up to $-46 \%$ ) in all serum-derived HDL subfractions from diabetic patients as compared with controls. This difference was, however, only significant for HDL3a (Table 2) particles. The distribution of PON1 activity between HDL subfractions was similar for diabetic and control subjects, specifically HDL $3 c>$ HDL 3b $>$ HDL 3a $>$ HDL2a $>$ HDL2b [23]. PAF-AH activity was significantly lower $(-18 \%)$ in the diabetic HDL3c subfraction as compared with controls (Table 2). LCAT activity tended to be higher in diabetic HDL subfractions as compared with controls; this difference was significant for HDL3b (Table 2) particles.

When parameters of LDL oxidation in the presence of HDL subfractions were correlated with activities of antioxidative proteins, PON1 activity was negatively correlated with the oxidation rate in the propagation phase and maximal amount of dienes, and positively correlated with the duration of lag and propagation phases (Table 3). Similarly, significant but weaker correlations were found for LCAT and PAF-AH activities (Table 3).

\section{Discussion}

Our present study has revealed for the first time that small dense HDL, specifically the $3 b$ and $3 c$ particle subfractions, in type 2 diabetic subjects under glycaemic control exhibit impaired capacity (up to $-52 \%$ ) to protect LDL from oxidative stress, both on a unit particle mass basis and on a particle number basis. Dysfunctional small dense HDLs displayed an abnormal chemical composition of both core (CE, TG) and surface (FC, PL) components, characteristic of the enrichment in TG and PL at the expense of $\mathrm{CE}$ and $\mathrm{FC}$, respectively, and tended to display diminished PON1 and PAF-AH activities. In both diabetic and non-diabetic groups, however, small dense HDL3 exerted more potent protection against LDL oxidation than large light HDL2, both on a unit mass basis and on a particle number basis. This observation was consistent with earlier studies in normolipidaemic subjects [23, 38, 39].

Oxidative stress plays a key role in the pathogenesis of the accelerated atherosclerosis characteristic of type 2 diabetes [3-6, 40, 41]. Indeed, the hyperglycaemia, hyperinsulinaemia and dyslipidaemia of type 2 diabetes are closely associated with increased generation of ROS and with endothelial dysfunction [3-6, 40, 41]. It is highly relevant therefore that systemic oxidative stress in our nonobese diabetic subjects under glycaemic control, assayed as plasma 8-isoprostane levels, was almost threefold higher than in controls, but in addition, was positively correlated with the degree of glycaemia and equally with a marker of an atherogenic lipid profile (TC:HDL-C ratio). In contrast, 8 -isoprostane concentrations correlated negatively with HDL-C. Moreover, 8-isoprostane levels were correlated with markers of LDL oxidation in the presence of HDL3b and $3 \mathrm{c}$, whereas fasting levels of glucose and TG were negatively correlated with sAA of HDL $3 b$ and $3 \mathrm{c}$ subfractions, respectively. Considered together, these observations attest to the functional relationships between glycaemia, dyslipidaemia, oxidative stress, and the capacity of HDL3 particles to protect LDL from oxidation in type 2 diabetes.

Elevated TG levels $(>1.76 \mathrm{mmol} / \mathrm{l}$, equivalent to 150 $\mathrm{mg} / \mathrm{dl}$ ) as found in our diabetic subjects are key determinants of the formation of small TG-rich HDL particles of altered metabolism [7, 20, 42, 43]. The diminished plasma residence time of TG-rich HDL in hypertriglyceridaemic diabetic subjects is a major factor accounting for their subnormal HDL-C levels [7, 42]. In such diabetic dyslipidaemia, which mechanisms may underlie the impaired antioxidative function of small HDL particle subfractions?

Several protein components of HDL particles possess antioxidative properties, including PON1, PAF-AH, LCAT, apoA-I, apoA-II and apoA-IV [9]. Recently, impaired PON1 activity was found to be closely linked to increased risk of cardiovascular mortality [44]. Moreover, PON1 activity is decreased in patients with type 2 diabetes without [45] or with [46] coronary artery disease. Furthermore, in vitro incubation with glucose of PON1-containing HDL or PON1 alone impairs PON1 activity [46]. In contrast, PON1 activity was normal in subjects with impaired glucose tolerance and with newly diagnosed type 2 diabetes [47], suggesting that impairment of PON1 activity occurs later in the course of the disease. Consistent with these findings, PON1 activity in our diabetic patients under glycaemic control was lower in all HDL subfractions; this difference was, however, only significant for HDL3a particles. Together with the finding that PON1 activity was strongly correlated with the SAA of HDL subfractions, these data suggest a role of PON1 in the impaired oxidative protection of LDL by diabetic HDL3 particles. On the other hand, PON1-independent inhibition of LDL oxidation by HDL has been previously demonstrated [23], indicating that components in addition to PON1 contribute significantly to the expression of potent antioxidative activity of small dense HDL. 
PAF-AH activity was significantly decreased in the diabetic HDL3c subfraction, suggesting that this enzyme might contribute to the impaired antioxidative activity of HDL3c particles in type 2 diabetes. In contrast, no reduction in LCAT activities was detectable in diabetic HDL subfractions, although PON1, PAF-AH and LCAT activities were each correlated with sAA of HDL subfractions. LCAT therefore constitutes a minor antioxidative component. Finally, apoA-I and apoA-II content of HDL subfractions did not differ between diabetic patients and controls, and in addition, did not correlate with sAA. Clearly then, on a quantitative basis, neither apoA-I nor apoA-II appear to account for the dysfunctional antioxidative activity of diabetic HDL3 subfractions.

In contrast, lipid components of HDL particles may influence antioxidative activity via both indirect and direct mechanisms. Enhanced CETP activity in type 2 diabetes leads to $\mathrm{CE}$ depletion and TG enrichment of the hydrophobic core of HDL particles [7, 12, 19, 41, 42], a finding confirmed in the present study. Replacement of CE by TG in the HDL lipid core results in reduced penetration of the central and C-terminal regions of apoA-I into the lipid phase [21]; such CE-lacking, TG-rich particles from diabetic subjects exhibit diminished acceptor capacity for cellular cholesterol $[14,15]$, consistent with the hypothesis that they may display attenuated capacity to accept oxidised CE, FC and/or PL (principally in the form of lipid hydroperoxides) from LDL [34]. Recently, we demonstrated that in vitro replacement of CE by TG in small dense HDL3 by incubation with VLDL in the presence of CETP abrogates HDL-mediated LDL protection from oxidation by AAPH (S. Chantepie, M. J. Chapman, A. Kontush, unpublished data). In this way, altered lipid core composition could potentially contribute significantly via an indirect mechanism to the attenuated antioxidative activity of diabetic HDL 3 particles, resulting in enhanced accumulation of lipid oxidation products in LDL under oxidative stress.

The deleterious effect of HDL core replacement of CE by TG on apoA-I function may be accentuated by hyperglycaemia, consistent with our observation that the degree of glycaemia was negatively correlated with sAA of HDL3 subfractions. Such findings may be accounted for by glycation of apoA-I, which exerts marked inhibition of its ability to facilitate cellular cholesterol efflux $[14,17]$. Glycation of apoA-I may, therefore, be paralleled by reduction in its antioxidative capacity when present as a component of HDL3 particles.

The deficient antioxidative activity of diabetic HDL3 subfractions may involve their minor content of lipophilic antioxidants, such as vitamin E [10]. It seems unlikely, however, that lipophilic antioxidants play an important role in our experimental system, since their amounts in HDL subfractions added to LDL are much lower than those needed to delay LDL oxidation [10,48]. In addition, dense HDL subfractions contain less lipophilic antioxidants per unit mass than light HDL [10], which contradicts the higher antioxidative activity of dense HDL3 vs light HDL2 sub- fractions [25]. Finally, no difference in plasma vitamin E levels was found between the study groups.

In contrast to our studies in well-controlled diabetic subjects, Gowri et al. [16] reported that HDL2, rather than HDL3, particles exhibited decreased protection against THP1 macrophage-mediated LDL oxidation in poorly controlled type 2 diabetes. These findings may be accounted for, at least in part (1) by an HDL2-specific attenuation of free radical production by THP-1 macrophages, and/or (2) by preferential glycation of apoA-I in HDL2 particles relative to that in the HDL3 subfraction at high glucose levels. Clearly, comparison of the capacity of HDL subfractions derived from well-controlled vs poorly controlled diabetic subjects to protect LDL against oxidation induced by different oxidative systems is needed to provide a mechanistic basis for the observed differences between these studies.

In conclusion, our studies reveal that the antioxidative dysfunctionality of small dense HDL3 particles in type 2 diabetes is closely associated with elevated oxidative stress and with the degree of glycaemia and triglyceridaemia. Such attenuated antioxidative activity may result from concomitant effects of abnormal lipid core composition, altered conformation and function of apoA-I and from decreased activities of HDL-associated enzymes, including PON1 and PAF-AH. Interestingly, plasma levels of HDL3 were a powerful predictor of cardiovascular risk in insulinresistant subjects in the VA-HIT trial [49]. Small dense HDL particles predominate in carriers of apoA-I Milano, who exhibit decreased cardiovascular risk [50]. In addition, infusion of recombinant small HDLs containing apoA-I Milano significantly decreased coronary atheroma volume in patients with acute coronary syndromes [51]. Clearly then, the normalisation of both levels and the anti-atherogenic function of small dense HDL3 particles in type 2 diabetes represents a key therapeutic target.

Acknowledgements These studies were supported by INSERM (Paris, France). E. Nobécourt was the recipient of a Research Fellowship from Nantes Centre University Hospital (CHU), and S. Chantepie was supported by the Association Claude Bernard. In addition, A. Kontush gratefully acknowledges the award of a Fellowship from the Foundation for Medical Research and an International HDL Research Award from Pfizer. We thank J.L. Beaudeux and D. Bonnefont-Rousselot for kindly measuring plasma CRP and vitamin E levels.

\section{References}

1. Haffner SM (2000) Coronary heart disease in patients with diabetes. N Engl J Med 342:1040-1042

2. Turner RC, Millns H, Neil HA et al (1998) Risk factors for coronary artery disease in non-insulin dependent diabetes mellitus: United Kingdom Prospective Diabetes Study (UKPDS: 23). Br Med J 316:823-828

3. Mezzetti A, Cipollone F, Cuccurullo F (2000) Oxidative stress and cardiovascular complications in diabetes: isoprostanes as new markers on an old paradigm. Cardiovasc Res 47:475-488 
4. Maxwell SR, Thomason H, Sandler D et al (1997) Antioxidant status in patients with uncomplicated insulin-dependent and non-insulin-dependent diabetes mellitus. Eur J Clin Invest 27:484-490

5. Wolff SP, Dean RT (1987) Glucose autoxidation and protein modification. The potential role of 'autoxidative glycosylation' in diabetes. Biochem J 245:243-250

6. Lusis AJ (2000) Atherosclerosis. Nature 407:233-241

7. Taskinen MR (2003) Diabetic dyslipidaemia: from basic research to clinical practice. Diabetologia 46:733-749

8. Barter P, Kastelein J, Nunn A, Hobbs R (2003) High density lipoproteins (HDLs) and atherosclerosis; the unanswered questions. Atherosclerosis 168:195-211

9. Navab M, Ananthramaiah GM, Reddy ST et al (2004) The oxidation hypothesis of atherogenesis: the role of oxidized phospholipids and HDL. J Lipid Res 45:993-1007

10. Goulinet S, Chapman MJ (1997) Plasma LDL and HDL subspecies are heterogenous in particle content of tocopherols and oxygenated and hydrocarbon carotenoids. Relevance to oxidative resistance and atherogenesis. Arterioscler Thromb Vasc Biol 17:786-796

11. Hasselwander O, McEneny J, McMaster D et al (1999) HDL composition and HDL antioxidant capacity in patients on regular haemodialysis. Atherosclerosis 143:125-133

12. Biesbroeck RC, Albers JJ, Wahl PW, Weinberg CR, Bassett ML, Bierman EL (1982) Abnormal composition of high density lipoproteins in non-insulin-dependent diabetics. Diabetes 31: $126-131$

13. Syvanne M, Ahola M, Lahdenpera S et al (1995) High density lipoprotein subfractions in non-insulin-dependent diabetes mellitus and coronary artery disease. J Lipid Res 36:573-582

14. Igau B, Castro G, Clavey V et al (1997) In vivo glucosylated LpA-I subfraction. Evidence for structural and functional alterations. Arterioscler Thromb Vasc Biol 17:2830-2836

15. Cavallero E, Brites F, Delfly B et al (1995) Abnormal reverse cholesterol transport in controlled type II diabetic patients. Studies on fasting and postprandial LpA-I particles. Arterioscler Thromb Vasc Biol 15:2130-2135

16. Gowri MS, Van der Westhuyzen DR, Bridges SR, Anderson JW (1999) Decreased protection by HDL from poorly controlled type 2 diabetic subjects against LDL oxidation may be due to the abnormal composition of HDL. Arterioscler Thromb Vasc Biol 19:2226-2233

17. Duell PB, Oram JF, Bierman EL (1991) Nonenzymatic glycosylation of HDL and impaired HDL-receptor-mediated cholesterol efflux. Diabetes 40:377-384

18. Julier K, Mackness MI, Dean JD, Durrington PN (1999) Susceptibility of low- and high-density lipoproteins from diabetic subjects to in vitro oxidative modification. Diabet Med $16: 415-423$

19. Scheffer PG, Bos G, Volwater HG, Dekker JM, Heine RJ, Teerlink T (2003) Associations of LDL size with in vitro oxidizability and plasma levels of in vivo oxidized LDL in type 2 diabetic patients. Diabet Med 20:563-567

20. Guerin M, Le Goff W, Lassel TS, Van Tol A, Steiner G, Chapman MJ (2001) Atherogenic role of elevated CE transfer from HDL to VLDL(1) and dense LDL in type 2 diabetes : impact of the degree of triglyceridemia. Arterioscler Thromb Vasc Biol 21:282-288

21. Curtiss LK, Bonnet DJ, Rye KA (2000) The conformation of apolipoprotein A-I in high-density lipoproteins is influenced by core lipid composition and particle size: a surface plasmon resonance study. Biochemistry 39:5712-572

22. Ashby DT, Rye KA, Clay MA, Vadas MA, Gamble JR, Barter PJ (1998) Factors influencing the ability of HDL to inhibit expression of vascular cell adhesion molecule-1 in endothelial cells. Arterioscler Thromb Vasc Biol 18:1450-1455

23. Kontush A, Chantepie S, Chapman MJ (2003) Small, dense HDL particles exert potent protection of atherogenic LDL against oxidative stress. Arterioscler Thromb Vasc Biol 23: $1881-1888$
24. Lindholm E, Agardh E, Tuomi T, Groop L, Agardh CD (2001) Classifying diabetes according to the new WHO clinical stages. Eur J Epidemiol 17:983-989

25. Kontush A, Hubner C, Finckh B, Kohlschutter A, Beisiegel U (1995) Antioxidative activity of ubiquinol-10 at physiologic concentrations in human low density lipoprotein. Biochim Biophys Acta 1258:177-187

26. Blackburn R, Giral P, Bruckert E et al (2001) Elevated Creactive protein constitutes an independent predictor of advanced carotid plaques in dyslipidemic subjects. Arterioscler Thromb Vasc Biol 21:1962-1968

27. Rumsey SC, Stucchi AF, Nicolosi RJ, Ginsberg HN, Ramakrishnan R, Deckelbaum RJ (1994) Human plasma LDL cryopreserved with sucrose maintains in vivo kinetics indistinguishable from freshly isolated human LDL in cynomolgus monkeys. J Lipid Res 35:1592-1598

28. Chapman MJ, Goldstein S, Lagrange D, Laplaud PM (1981) A density gradient ultracentrifugal procedure for the isolation of the major lipoprotein classes from human serum. J Lipid Res $22: 339-358$

29. Guerin M, Le Goff W, Frisdal E et al (2003) Action of ciprofibrate in type IIb hyperlipoproteinemia: modulation of the atherogenic lipoprotein phenotype and stimulation of highdensity lipoprotein-mediated cellular cholesterol efflux. J Clin Endocrinol Metab 88:3738-3746

30. Guerin M, Lassel TS, Le Goff W, Farnier M, Chapman MJ (2000) Action of atorvastatin in combined hyperlipidemia: preferential reduction of cholesteryl ester transfer from HDL to VLDL1 particles. Arterioscler Thromb Vasc Biol 20:189-197

31. Chancharme L, Therond P, Nigon F, Lepage S, Couturier M, Chapman MJ (1999) Cholesteryl ester hydroperoxide lability is a key feature of the oxidative susceptibility of small, dense LDL. Arterioscler Thromb Vasc Biol 19:810-820

32. Steinbrecher UP, Pritchard PH (1989) Hydrolysis of phosphatidylcholine during LDL oxidation is mediated by plateletactivating factor acetylhydrolase. J Lipid Res 30:305-315

33. Wiltshire T, Pletcher MT, Batalov S et al (2003) Genome-wide single-nucleotide polymorphism analysis defines haplotype patterns in mouse. Proc Natl Acad Sci U S A 100:3380-3385

34. Kontush A, de Faria EC, Chantepie S, Chapman MJ (2004) Antioxidative activity of HDL particle subspecies is impaired in hyperalphalipoproteinemia: relevance of enzymatic and physicochemical properties. Arterioscler Thromb Vasc Biol 24:526533

35. Mol MJ, de Rijke YB, Demacker PN, Stalenhoef AF (1997) Plasma levels of lipid and cholesterol oxidation products and cytokines in diabetes mellitus and cigarette smoking: effects of vitamin E treatment. Atherosclerosis 129:169-176

36. Leinonen JS, Rantalaiho V, Solakivi T et al (1998) Susceptibility of LDL to oxidation is not associated with the presence of coronary heart disease or renal dysfunction in NIDDM patients. Clin Chim Acta 275:163-174

37. Devaraj S, Hirany SV, Burk RF, Jialal I (2001) Divergence between LDL oxidative susceptibility and urinary $\mathrm{F}(2)$-isoprostanes as measures of oxidative stress in type 2 diabetes. Clin Chem 47:1974-1979

38. Yoshikawa M, Sakuma N, Hibino T, Sato T, Fujinami T (1997) HDL3 exerts more powerful anti-oxidative, protective effects against copper-catalyzed LDL oxidation than HDL2. Clin Biochem 30:221-225

39. Huang JM, Huang ZX, Zhu W (1998) Mechanism of highdensity lipoprotein subfractions inhibiting copper-catalyzed oxidation of low-density lipoprotein. Clin Biochem 31:537-543

40. Cai H, Harrison DG (2000) Endothelial dysfunction in cardiovascular diseases: the role of oxidant stress. Circ Res 87: 840844

41. Arcaro G, Cretti A, Balzano S et al (2002) Insulin causes endothelial dysfunction in humans: sites and mechanisms. Circulation 105:576-582

42. Rashid S, Uffelman KD, Lewis GF (2002) The mechanism of HDL lowering in hypertriglyceridemic, insulin-resistant states. J Diabetes Complications 16:24-28 
43. Borggreve SE, De Vries R, Dullaart RP (2003) Alterations in high-density lipoprotein metabolism and reverse cholesterol transport in insulin resistance and type 2 diabetes mellitus: role of lipolytic enzymes, lecithin: cholesterol acyltransferase and lipid transfer proteins. Eur J Clin Invest 33:1051-1069

44. Mackness B, Durrington P, McElduff P et al (2003) Low paraoxonase activity predicts coronary events in the Caerphilly Prospective Study. Circulation 107:2775-2779

45. Abbott CA, Mackness MI, Kumar S, Boulton AJ, Durrington PN (1995) Serum paraoxonase activity, concentration, and phenotype distribution in diabetes mellitus and its relationship to serum lipids and lipoproteins. Arterioscler Thromb Vasc Biol 15:1812-1818

46. Hedrick CC, Thorpe SR, Fu MX et al (2000) Glycation impairs high-density lipoprotein function. Diabetologia 43:312-320

47. Kopprasch S, Pietzsch J, Kuhlisch E, Graessler J (2003) Lack of association between serum paraoxonase 1 activities and increased oxidized low-density lipoprotein levels in impaired glucose tolerance and newly diagnosed diabetes mellitus. J Clin Endocrinol Metab 88:1711-1716
48. Dieber-Rotheneder M, Puhl H, Waeg G, Striegl G, Esterbauer H (1991) Effect of oral supplementation with D-alpha-tocopherol on the vitamin E content of human low density lipoproteins and resistance to oxidation. J Lipid Res 32:1325-1332

49. Robins SJ, Collins D, Wittes JT et al (2001) Relation of gemfibrozil treatment and lipid levels with major coronary events: VA-HIT: a randomized controlled trial. JAMA 285: $1585-1591$

50. Chiesa G, Sirtori CR (2003) Apolipoprotein A-I(Milano): current perspectives. Curr Opin Lipidol 14:159-163

51. Nissen SE, Tsunoda T, Tuzcu EM et al (2003) Effect of recombinant ApoA-I Milano on coronary atherosclerosis in patients with acute coronary syndromes: a randomized controlled trial. JAMA 290:2292-2300 